\title{
Analysing Trends of Guillain-Barre Syndrome (GBS) and Dengue cases in Hong Kong
}

\section{Xin Wang*}

Shenzhen Center for Disease Control and Prevention, Shenzhen, China

\section{Objective}

To study the trends of GBS and dengue in Hong Kong, the ecological associations between GBS, dengue, and local meteorological factors. To examine the non-stationary oscillating association among these factors.

\section{Introduction}

Guillain-Barre Syndrome (GBS) is a severe paralytic neuropathy associated with virus infections such as Zika virus and Chikungunya virus. There were also case reports of dengue fever preceding GBS. With the aim to understand the mechanisms of GBS and dengue outbreaks, this ecological study investigates the relationships between GBS, dengue, meteorological factors in Hong Kong and global climatic factors from January 2000 to June 2016.

\section{Methods}

The correlations between GBS, dengue, Multivariate El Nino Southern Oscillation Index (MEI) and local meteorological data were explored by Spearman's Rank correlations and cross-correlations. Three Poisson regression models were fitted to identify non-linear associations among GBS, dengue and MEI. Cross wavelet analyses were applied to infer potential non-stationary oscillating associations among GBS, dengue and MEI.

\section{Results}

We found a substantial increasing of local GBS and dengue cases (mainly imported) in recent year in Hong Kong. The seasonalities of GBS and dengue are different, in particular, GBS is low while dengue is high in the summer. We observed weak but significant correlations between GBS and local meteorological factors. MEI could explain over $17 \%$ of dengue's variations based on Poisson regression analyses. We report a possible non-stationary oscillating association between dengue fever and GBS cases in Hong Kong.

\section{Conclusions}

We report increasing patterns of both local GBS cases and imported dengue cases in Hong Kong, and investigate the possible mechanism behind these patterns. This study has led to an improved understanding about the timing and ecological relationships between MEI, GBS and dengue.

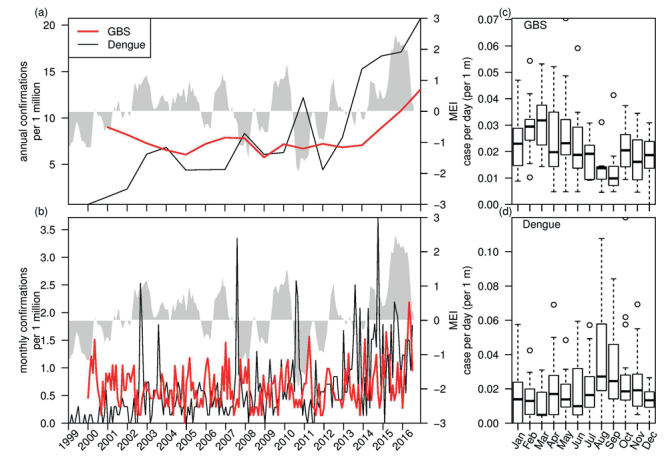

Fig 1. Trends and seasonality of GBS and dengue cases (scaled by number of population in Hong Kong). Panel (a), Annual cases of GBS and dengue cases show a sudden increase in recent years. Panel (b), Monthly cases of GBS and dengue cases. The grey shaded area of panel $(\mathrm{a}, \mathrm{b})$ is MEI. Panel (c), Boxplot of GBS cases per day. Panel (d), Boxplot of dengue cases per day.
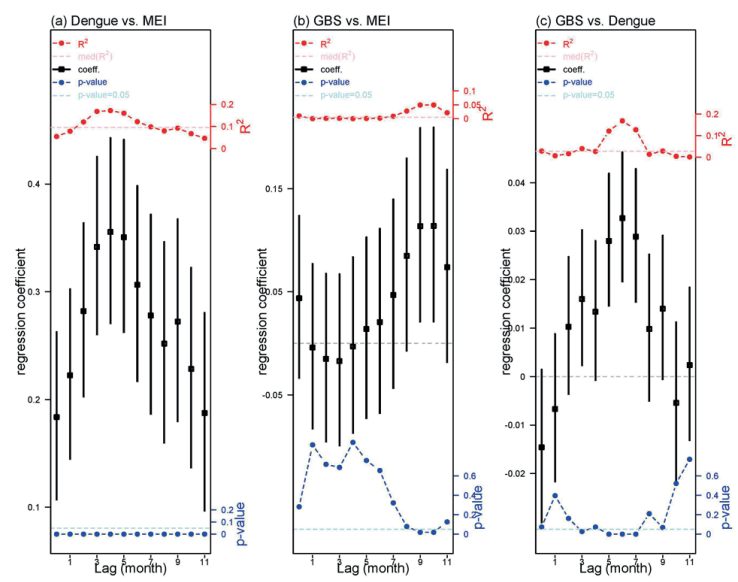

Fig 2. Poisson regression results among dengue, MEI and GBS. Panel (a) shows regression coefficients between dengue and MEI, panel (b) shows regression coefficients between GBS and MEI and panel (c) shows regression coefficients between GBS and dengue. In all three panels, we consider time lags from 0 to 11 months. The vertical black bars are 95\% confidence intervals and the squares in the middle are the mean estimate of regression coefficients. The blue dotted line is p-value of each correlation coefficient. The horizontal dashed light blue lines on all panels indicate the 0.05 significance level. The red dotted line is R2 of each regression coefficient. The horizontal dashed pink lines represent the median level of all R2.
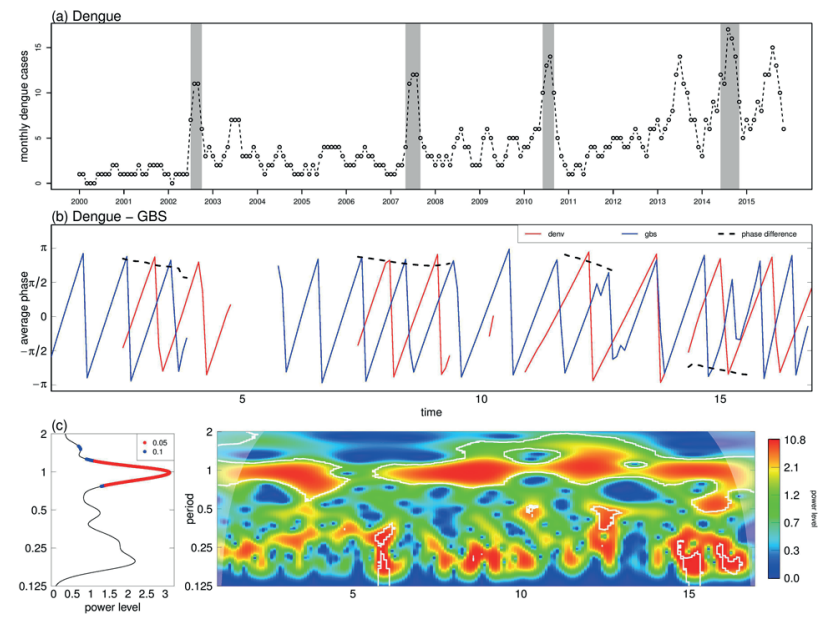

Fig 3. Wavelet coherence and phase plots of dengue and GBS data from 2000-15 in Hong Kong. Panel (a) is dengue time series with peaks shaded in grey. Panel (b) are phase plots of dengue and GBS. Data are shown in red and blue, and the black dashed line shows phase difference. Panel (c) shows cross wavelet average power level and wavelet coherence plots of dengue and GBS. The horizontal axis labels of 5, 10 and 15 represent year 2005, 2010 and 2015.

\section{Keywords}

Guillain-Barre Syndrome; Dengue; survaillance

\section{*Xin Wang}

E-mail: szwxin@163.com 